\title{
Matter density perturbations in modified gravity models with arbitrary coupling between matter and geometry.
}

\author{
Savvas Nesseris* $*$ \\ The Niels Bohr International Academy, The Niels Bohr Institute, 2100 Copenhagen Ø, Denmark
}

(Dated: April 10, 2018)

\begin{abstract}
We consider theories with an arbitrary coupling between matter and gravity and obtain the perturbation equation of matter on subhorizon scales. Also, we derive the effective gravitational constant $G_{e f f}$ and two parameters $\Sigma$ and $\eta$, which along with the perturbation equation of the matter density are useful to constrain the theory from growth factor and weak lensing observations. Finally, we use a completely solvable toy model which exhibits nontrivial phenomenology to investigate specific features of the theory. We obtain the analytic solution of the modified Friedmann equation for the scale factor $a$ in terms of time $t$ and use the age of the oldest star clusters and the primordial nucleosynthesis bounds in order to constrain the parameters of our toy model.
\end{abstract}

PACS numbers: 04.50.Kd,95.30.Sf,98.80.-k

\section{INTRODUCTION}

There is accumulating observational evidence based mainly on type Ia supernovae standard candles [1] that the universe has entered a phase of accelerating expansion at a recent cosmological time scale. This expansion implies the existence of a repulsive factor on cosmological scales which counterbalances the attractive gravitational properties of matter on these scales. There have been several theoretical approaches [2], [3] towards the understanding of the origin of this factor. The simplest such approach assumes the existence of a positive cosmological constant which is small enough to have started dominating the universe at recent times. This model provides an excellent fit to the cosmological observational data [4] and has the additional bonus of simplicity and a single free parameter. Despite its simplicity and good fit to the data, this model fails to explain why the cosmological constant is so unnaturally small as to come to dominate the universe at recent cosmological times, a problem known as the coincidence problem and there are specific cosmological observations which differ from its predictions [5], 6].

In an effort to address this problem two classes of models have been proposed: The first class assumes that general relativity (GR) is a valid theory on cosmological scales and attributes the accelerating expansion to a dark energy component which has repulsive gravitational properties due to its negative pressure. The role of dark energy is usually played by a minimally coupled to gravity scalar field called quintessence 7]. Alternatively, the role of dark energy can be played by various perfect fluids (e.g., Chaplygin gas [8]), topological defects 9], holographic dark energy [10], etc. The second class of models attributes the accelerating expansion to a modification of general relativity on cosmological scales which converts gravity to a repulsive interaction at late times and on cos-

*Electronic address: nesseris@nbi.dk mological scales. Examples of this class of models include scalar-tensor theories [11, 12], 13], $f(R)$ modified gravity theories 14], braneworld models [15], etc.

Of these examples, $f(R)$ theories have received much attention mainly due to the fact that they can provide a more natural explanation of the accelerating expansion of the Universe. At the same time many of these are able to satisfy the solar system constraints, see, for example, Ref. [16], and this is done without the introduction of extra scalar field degrees of freedom, for a review of viable $f(R)$ gravity models see [17], [18], while for the advantages and the drawbacks of this class of theories see [19] and [20]. Also, the $f(R)$ theories arise in a wide range of different frameworks: In quantum field theories in curved spacetime [21], in the low energy limit of the $D=10 \mathrm{su}-$ perstring theory 22], in the vacuum action for the grand unified theories, etc.

Recently another approach was proposed, first in [23], [24] and later in [25], namely, to add as a nonminimal coupling to matter a general function of the Ricci scalar. Such nonlinear couplings of matter were analyzed in the past [26] in the context of the accelerated expansion of the Universe and in Refs. 27], 28] it was shown that these theories do not correspond to scalar-tensor gravity and therefore have a yet unexplored and non-trivial phenomenology. Furthermore, such theories due to the explicit nonminimal coupling between the matter content and gravity have a nonvanishing covariant divergence of the matter energy-momentum tensor $T_{\mu \nu}$ [29], 30], [31], which means that there is an energy exchange between matter and gravity.

In Sec. II we will derive the background equations of motion for a general Lagrangian density $\frac{1}{2} f_{1}(R)+$ $G\left(L_{m}\right) f_{2}(R)$ and we will discuss some implications about the possible choices for the matter Lagrangian $L_{m}$. Having more than one Lagrangians to describe the same fluid, e.g. $L_{m}=p$ or $L_{m}=-\rho$ for pressureless dust, is not a problem in GR as all choices will give the same equations of motion, but in theories with a nonminimal coupling, different choices in general will give different phenomenology. Nevertheless, we will choose a Lagrangian 
that depends only on the density $L_{m}=L_{m}\left(\rho_{m}\right)$ in order to keep the analysis as simple as possible, but quite general at the same time.

In Sec. III we will derive the matter density perturbation equations for the case that the matter Lagrangian is a general function of the density $\rho$. Being able to predict the evolution of the matter perturbations for such modified gravity models is necessary in order to compare the theory against the linear growth data. Such data are in principle able to differentiate between GR and modified gravity, with the main reasons being the evolution with time of the gravitational constant [32], [33].

We will also evaluate two parameters $\eta$ and $\Sigma=q(1+$ $\eta / 2$ ) for this particular model, following Refs [34, 35] where the first quantifies the strength of an anisotropic stress while in the latter, $q$ quantifies the deviation of the gravitational constant today. These parameters can be used to constrain the theory with weak lensing observations and if such surveys measure deviations from GR, for which $\left.(\Sigma, \eta)\right|_{G R}=(1,0)$ then those two parameters could be used to differentiate between modified gravity and dark energy models.

Finally, in Sec. IV we will use a toy model with Lagrangian density $R / 2+(1+\lambda R) G\left(L_{m}\right)$, where $G\left(L_{m}\right)$ is given by $G\left(L_{m}\right)=A L_{m}^{n+1}$ and $L_{m}=-\rho$. This model has the energy-momentum tensor of pressureless dust, but exhibits nontrivial phenomenology compared to its counterpart from GR. Also, we will present an analytical solution to the modified Friedmann equation and give the scale factor $a$ in terms of the cosmic time $t$. In order to provide some bounds on the parameters $\lambda$ and $n$ of our toy model, we will use the following two observations: the age of the oldest star clusters and the Big Bang Nucleosynthesis (BBN) bounds on the gravitational constant $G_{N}$, which are of the order of $10 \%$.

\section{BACKGROUND EQUATIONS}

The action we will consider is

$$
S=\int d^{4} x \sqrt{-g}\left[\frac{1}{2} f_{1}(R)+G\left(L_{m}\right) f_{2}(R)\right]
$$

where $g$ is the determinant of the metric $g_{\mu \nu}$ and $f_{i}=$ $f_{i}(R), i=1,2$ are arbitrary functions of the Ricci scalar $R$, while $G\left(L_{m}\right)$ is an arbitrary function of the matter Lagrangian density $L_{m}$. We use the metric signature $(-,+,+,+)$.

Varying the action with respect to the metric $g_{\mu \nu}$ we obtain the field equations as

$$
\begin{gathered}
{\left[F_{1}(R)+2 G\left(L_{m}\right) F_{2}(R)\right] R_{\mu \nu}-\frac{1}{2} f_{1}(R) g_{\mu \nu}+} \\
+\left[g_{\mu \nu} \square-\nabla_{\mu} \nabla_{\nu}\right]\left(F_{1}(R)+2 G\left(L_{m}\right) F_{2}(R)\right)- \\
-g_{\mu \nu} f_{2}(R)\left[G\left(L_{m}\right)-K\left(L_{m}\right) L_{m}\right]= \\
=f_{2}(R) K\left(L_{m}\right) T_{\mu \nu}
\end{gathered}
$$

where $F_{i}=\partial f_{i} / \partial R, i=1,2, R_{\mu \nu}$ is the Ricci tensor and $K\left(L_{m}\right)=\partial G\left(L_{m}\right) / \partial L_{m}$. When $f_{1}(R)=f(R), f_{2}(R)=$
1 and $G\left(L_{m}\right)=L_{m}$ then we recover the field equations for $f(R)$ gravity.

Finally, we have defined the energy-momentum tensor of the matter as

$$
T_{\mu \nu}=-\frac{2}{\sqrt{-g}} \frac{\delta\left(\sqrt{-g} L_{m}\right)}{\delta g^{\mu \nu}}
$$

Assuming that the matter Lagrangian density $L_{m}$ depends only on the metric and not on it's components, we obtain $T_{\mu \nu}=L_{m} g_{\mu \nu}-2 \partial L_{m} / \partial g^{\mu \nu}$. In what follows we will assume that the matter Lagrangian is an arbitrary function of the energy density $\rho$ only, so that $L_{m}=L_{m}(\rho)$. Then the energy-momentum tensor can be written as

$$
T^{\mu \nu}=-\rho \frac{d L_{m}}{d \rho} u^{\mu} u^{\nu}+\left(L_{m}-\rho \frac{d L_{m}}{d \rho}\right) g^{\mu \nu}
$$

where the four-velocity $u^{\mu}=d x^{\mu} / d s$ satisfies the condition $u^{\mu} u_{\mu}=-1$. In deriving Eq.(2.4) we have used Eq.(2.3), the relation $\delta \rho=\frac{1}{2} \rho\left(g_{\mu \nu}+u_{\mu} u_{\nu}\right) \delta g^{\mu \nu}$ and the conservation of the matter current $\nabla_{\nu}\left(\rho u^{\nu}\right)=0$. Note that the difference in the sign of the first term between our Eq.(2.4) and the corresponding one of Ref. 31] is due to the difference in the normalization condition, $u^{\mu} u_{\mu}=-1$ for us.

In general the Lagrangian of a perfect fluid (on shell) is equal to the pressure $L_{m}=p$, ie for dust it is equal to zero. However, this is not the only choice as the Lagrangian $L_{m}=-\rho$ also reproduces the same energymomentum tensor $T_{\mu \nu}=\rho u_{\mu} u_{\nu}$. This is not a problem in GR as both choices give the same equations of motion, but in theories with a nonminimal coupling, different choices give different phenomenology. This might be a problem since our choice for the Lagrangian becomes physically meaningful without having any criteria for choosing it, besides the observations of course.

This fact has created some debate on the literature lately [27], 29], 230] regarding which is is the best choice for the Lagrangian $L_{m}$ for the perfect fluid. Nevertheless, we choose a Lagrangian that depends only on density $L_{m}=L_{m}\left(\rho_{m}\right)$ in order to keep the analysis as simple as possible but quite general at the same time.

In a flat Friedmann-Robertson-Walker metric with a scale factor $a(t)$, we obtain the background (zero-order) equations:

$$
\begin{gathered}
3\left[F_{1}+2 G F_{2}\right] H^{2}=f_{2} K \rho_{m}+\frac{1}{2}\left[\left(F_{1}+2 G F_{2}\right) R-f_{1}\right] \\
-f_{2}\left(G-K L_{m}\right)-3 H \frac{d}{d t}\left(F_{1}+2 G F_{2}\right) \\
\quad-2\left[F_{1}+2 G F_{2}\right] \dot{H}=f_{2} K \rho_{m}+ \\
+\left(\frac{d^{2}}{d t^{2}}-H \frac{d}{d t}\right)\left[F_{1}+2 G F_{2}\right]
\end{gathered}
$$

where $H=\frac{\dot{a}}{a}, R=6\left(2 H^{2}+\dot{H}\right)$ and a dot represents a derivative with respect to the cosmic time t. 
In order to get the evolution equation for the ideal fluid we take the covariant divergence of Eq. (2.2) and by using the purely geometrical identities [36]: $\nabla^{\mu} G_{\mu \nu}=0$ and $\left(\square \nabla_{\nu}-\nabla_{\nu} \square\right) F=R_{\mu \nu} \nabla^{\mu} F$ we get 31]

$$
\nabla^{\mu} T_{\mu \nu}=\left(g_{\mu \nu} L_{m}-T_{\mu \nu}\right) \nabla^{\mu} \ln \left(f_{2} K\right)
$$

For the case at hand (2.7) gives

$$
\dot{\rho}_{m}=\frac{-3 K\left[f_{2} H \rho_{m}+2 F_{2}\left(L_{m}+\rho_{m}\right)(4 H \dot{H}+\ddot{H})\right]}{f_{2}\left(K+\left(L_{m}+\rho_{m}\right) K^{\prime}\left(L_{m}\right) L_{m}^{\prime}\left(\rho_{m}\right)\right)}(2.8)
$$

where the prime denotes differentiation with respect to the particular argument, i.e., $K^{\prime}\left(L_{m}\right)=\partial K / \partial L_{m}$ while $L_{m}^{\prime}\left(\rho_{m}\right)=\partial L_{m} / \partial \rho_{m}$. Obviously, when $G=L_{m}$ and $f_{2}(R)=1$ we recover the usual energy conservation equation of GR and $f(R)$ theories.

\section{PERTURBATION EQUATIONS}

We will consider the following perturbed metric with scalar metric perturbations $\Phi$ and $\Psi$ in a longitudinal gauge:

$$
d s^{2}=-(1+2 \Phi) d t^{2}+a(t)^{2}(1-2 \Psi) \delta_{i j} d x^{i} d x^{j}
$$

The energy-momentum tensor of the nonrelativistic matter is decomposed as $T_{0}^{0}=-\left(\rho_{m}+\delta \rho_{m}\right)$ and $T_{\alpha}^{0}=$ $-\rho_{m} v_{m, \alpha}$, where $v_{m}$ is a velocity potential. The Fourier transformed perturbation equations are given in the Appendix by Eqs. (A1), (A2), (A3) and (A4).

Next, we define the gauge invariant matter density perturbation $\delta_{m}$ as

$$
\delta_{m} \equiv \frac{\delta \rho_{m}}{\rho_{m}}+3 H v
$$

where

$$
v=a v_{m}
$$

Following the approach of Ref. [35], we use a subhorizon approximation under which the leading terms correspond to those containing $k^{2}$ and $\delta \rho_{m}$. Terms that are of the form $H^{2} \Phi$ or $\ddot{\Phi}$ are considered negligible relative to terms like $\left(k^{2} / a^{2}\right) \Phi$ for modes well inside the Hubble radius $\left(k^{2} \gg a^{2} H^{2}\right)$. Under this approximation Eqs. (A3), (A4) and (3.2) yield a second order differential equation for $\delta$, given in the Appendix by Eq. A5).

Equation (A5) compared to the corresponding one of GR or $f(R)$ theories (see for example [35], 37], 38]) has an extra term which is a function of the matter content of the theory. This fact, as we will see in what follows, will force Newton's constant $G_{e f f}$ to be dependent on the matter content, something which is in sharp contrast with $f(R)$ theories, but not scalar-tensor gravity where $G_{\text {eff }}$ may depend on the scalar field $\phi$ through it's nonminimal coupling $F(\phi)$.
Next, we write $\delta F_{1}$ and $\delta F_{2}$ as

$$
\delta F_{i}=F_{i, R} \delta R
$$

where $i=1,2$, the comma denotes derivative with respect to the Ricci scalar $R$ and $\delta R$, under the subhorizon approximation, is given by:

$$
\delta R \simeq-2 \frac{k^{2}}{a^{2}}(2 \Psi-\Phi)
$$

Making these substitutions and using the subhorizon approximation in Eqs. (A1) and (A2) we get a set of equations for $\Phi$ and $\Psi$ given by Eqs. (A6) and (A7). The next step is to express $\Phi$ and $\Psi$ in terms of $\delta_{m}$. This can be done by solving the system (A6) and (A7) for $\Phi$ and $\Psi$. Doing so we find $\Phi$ and $\Psi$ under the subhorizon approximation, given by Eqs. (A8) and (A9).

From the equation for $\Phi$ (A9) we can define a Poisson equation in the Fourier space and attribute the extra terms that appear on the right-hand side to an effective gravitational constant $G_{e f f}$. Doing so, we get the gravitational potential

$$
\Phi=-4 \pi G_{e f f} \frac{a^{2}}{k^{2}} \delta_{m} \rho_{m}
$$

where $G_{\text {eff }}$ is given by (A10).

Hence, the equation of matter perturbations (A5) can be written compactly as

$$
\ddot{\delta}_{m}+2 H \dot{\delta}_{m}-4 \pi\left(G_{e f f}+\beta\right) \rho_{m} \delta_{m} \simeq 0
$$

where the parameter $\beta$, after using Eqs. (A8) and (A9), is given by (A11). As it can be seen from Eq. (A11) in the minimal coupling case $\left[f_{2}(R)=1\right.$ and $\left.G\left(L_{m}\right)=L_{m}\right]$ we get the expected result $\beta=0$.

We also define two parameters $\eta$, which characterizes the strength of the anisotropic stress

$$
\eta \equiv \frac{\Phi-\Psi}{\Psi}
$$

and $q$ via $\frac{k^{2}}{a^{2}} \Psi=-4 \pi G_{0} q \rho_{m} \delta_{m}$, where $G_{0}$ is the value of the gravitational constant measured by the solar system experiments today. Then these two parameters are given by Eqs (A12) and (A13).

In order to confront the modified gravity models with weak lensing observations it is necessary to calculate the potential $\Phi_{W L}$ that characterizes the deviation of light rays. Under the assumptions that photons follow null geodesics and that the geodesic deviation equation holds, the lensing potential is $\Phi_{W L} \simeq \Phi+\Psi$; see Ref. 39] for a quick derivation and a discussion. However, in theories with nonminimal coupling to matter this might not always be the case. In Ref. [27] it was shown that for theories with Lagrangian $f_{1}(R) / 2+\left(1+\lambda f_{2}(R)\right) L_{m}$, nonminimal coupling corrections to the Maxwell equations which would otherwise affect standard optics, can actually affect only long, compared to the radius of the curvature of spacetime, wavelengths. When this is not the 
case, i.e. we consider the high-frequency limit, photons are transverse and propagate along null geodesics.

Having this in mind, we define a combination of parameters $\Sigma=q(1+\eta / 2)$, for which

$$
\Phi_{W L} \simeq \Phi+\Psi=-8 \pi G_{0} \frac{a^{2}}{k^{2}} \rho_{m} \delta_{m} \Sigma
$$

where the explicit form of $\Sigma$ is given by Eq. (A14). Again, in the case of the minimal coupling $\left[f_{2}(R)=1\right.$ and $\left.G\left(L_{m}\right)=L_{m}\right]$ we get the expected result for $\Sigma$ :

$$
\Sigma_{f_{R}}=\frac{1}{8 \pi F G_{0}}
$$

which agrees with the result of Refs. [35], 34] for simple $f(R)$ theories. We should remind the reader here that the formulas derived for the weak lensing potential have been derived under the assumption that light travels in null geodesics, which may be the case only for certain models and in the high-frequency limit.

Therefore, this class of theories may have non-trivial effects on the weak lensing that could potentially be observable. However, this would also require one to work out the most general case for the relation between the lensing potential and the metric perturbations. Since this is beyond the scope of this analysis, we have left the most general case for future work.

In order to recover $f(R)$ gravity for all the results we have mentioned, we only have to set $f_{1}(R)=f(R)$, $f_{2}(R)=1$, and $G\left(L_{m}\right)=L_{m}$. This means that $F_{2}(R)=$ $0, K\left(L_{m}\right)=1$, and $K^{\prime}\left(L_{m}\right)=0$. Then, for example, Eq. (A10) yields

$$
G_{e f f}=\frac{1}{8 \pi F} \frac{1+4 \frac{k^{2}}{a^{2} R} m}{1+3 \frac{k^{2}}{a^{2} R} m}
$$

where

$$
m \equiv \frac{R F_{, R}}{F}
$$

also the anisotropic parameter $\eta$ defined in (3.8) and given by (A12) becomes

$$
\eta=\frac{2 \frac{k^{2}}{a^{2} R} m}{1+2 \frac{k^{2}}{a^{2} R} m}
$$

with both equations, (3.11) and (3.12), being in agreement with the standard results from $f(R)$ gravity [35].

\section{A TOY MODEL}

As an example we will now consider the case where we have the Lagrangian of GR plus a specific nonminimal coupling of gravity to pressureless dust. Specifically, for our toy model we will assume that $f_{1}(R)=R$, $f_{2}(R)=1+\lambda R, L_{m}(\rho)=-\rho$ and $G\left(L_{m}\right)=A L_{m}^{1+n}$, where $\lambda, A$ and $n$ are constants. From Eq. (2.4) we see that the energy-momentum tensor then is given by $T_{\mu \nu}=\rho u_{\mu} u_{\nu}$ as usual. The modified Friedmann and continuity equations can easily be found from Eqs. (2.5) and (2.8). For the continuity equation we find

$$
3 \Omega(a)+a \Omega^{\prime}(a)=0
$$

where the derivatives are written in terms of the scale factor $a$ and we have defined $\rho(a) \equiv 3 H_{0}^{2} \Omega(a)$.

Equation (4.1) can as usual be solved for $\Omega(a)$ and with initial conditions $\Omega(1)=1$ we get

$$
\Omega(a)=\frac{1}{a^{3}}
$$

For the modified Friedmann we find, after using (4.2)

$$
H(a)^{2}=\frac{(-3)^{n} H_{0}^{2 n+2} A}{2(-1)^{n} 3^{n+1} A(3 n+2) \lambda H_{0}^{2 n+2}+a^{3 n+3}}
$$

By demanding that $H(1) \equiv H_{0}$ we can solve for $A$ and after substituting back to the previous equation we find

$$
H(a)^{2}=\frac{H_{0}^{2}}{a^{3 n+3}\left(1-6 H_{0}^{2}(3 n+2) \lambda\right)+6 H_{0}^{2}(3 n+2) \lambda}(4.4)
$$

Obviously, when $\lambda=0$ and $n=0$ we get the usual, from GR, Friedmann equation for a matter dominated universe. If we expand (4.4) for $\lambda \ll 1$ we get

$$
\begin{aligned}
& H(a)^{2} \simeq H_{0}^{2}\left[a^{-3(n+1)}+\right. \\
& \left.6 a^{-6(n+1)}\left(a^{3 n+3}-1\right) H_{0}^{2}(3 n+2) \lambda+O\left(\lambda^{2}\right)\right]
\end{aligned}
$$

Thus, when $\lambda \ll 1$ such models can be thought of as a sum of ideal fluids with the first term being attributed to a matter fluid component with constant equation of state $w=n$, while the second to a dark energy fluid with variable equation of state $w=w(z)$.

We can use Eq.(4.4) to solve for $a(t)$ in terms of the cosmic time $t$ with the initial condition $a\left(t_{0}\right)=1$, where $t_{0}$ is the present time (the age of the universe). Doing so we find $a(t)$ in implicit form:

$$
t=\frac{a(t)^{3 n+3} m_{3} F_{2}\left(\frac{1}{2}, 1,1 ; 2,2 ;-a(t)^{3 n+3} m\right)}{\sqrt{m+1}(6 n+6)}
$$

where $m=\frac{1-6 H_{0}^{2}(3 n+2) \lambda}{6 H_{0}^{2}(3 n+2) \lambda}$ and ${ }_{3} F_{2}$ is a hypergeometric function. Then the age of the universe is

$$
t(a=1) \equiv t_{0}=\frac{m_{3} F_{2}\left(\frac{1}{2}, 1,1 ; 2,2 ;-m\right)}{6 H_{0} \sqrt{m+1}(n+1)}
$$

Demanding that the universe should be at least old enough to accommodate the oldest star clusters [40], then it is necessary to have $t_{0}>12$ Gyr. Equation (4.7) also implies that $\lambda$ and $n$ should satisfy certain conditions in order to have a real value for $t_{0}$. Specifically, we find that these conditions are $\lambda>0$ and $n>-\frac{2}{3}$ or $\lambda<0$ 


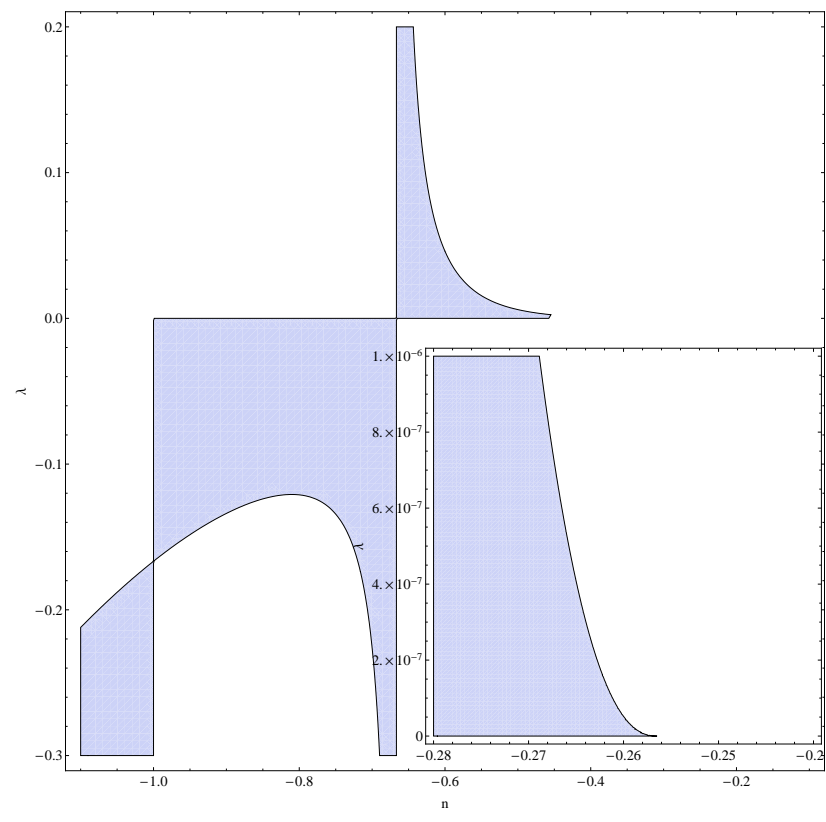

FIG. 1: The allowed parameter space of $(n, \lambda)$ such that $t_{0}>$ $12 G y r$, where $\lambda$ is in units of $1 / H_{0}^{2}$. The embedded plot shows the region around $\lambda \ll 1$ near the upper bound for $n$.

and $n<-\frac{2}{3}$. In Fig. 1 we show the allowed parameter space of $(n, \lambda)$ such that $t_{0}>12 G y r$. As it can be seen from the embedded plot there is an upper limit to $n$ that corresponds to $\lambda=0$ and $t_{0}=\frac{2}{3(n+1) H_{0}}$, with the latter giving $n<-0.256$ after we use the bound $t_{0}>12 G y r$.

Next we will use the primordial nucleosynthesis (BBN) constraints on the variation of the gravitational constant to further constrain the parameters $\lambda$ and $n$. The effect of the variation of $G_{\text {eff }}$ can be constrained from BBN to be of the order of $10 \%$, see for example Ref. [41] which gives $\frac{G_{B B N}}{G_{0}}=1.09 \pm_{0.19}^{0.22}$. The effective gravitational constant $G_{\text {eff }}$ can be calculated from (A10) by using (4.2) and (4.4). If we set $G_{e f f}(a=1) \equiv G_{0}$ then the gravitational constant is given by Eq. A15.

First, we will consider the following two cases, for $\lambda=0$ and $n=0$. In the first case the gravitational constant is simply given by $G_{e f f}=G_{0} a^{-3 n}$ and by using the BBN constraint of Ref. [41] we find $-0.0018<n<0.0045$ while in the second case by using a series expansion for small $\lambda$, which is to be expected from the Solar System tests, we find

$G_{e f f} / G_{0}=1+\left(2 k^{2} / H_{0}^{2}+\frac{9-2 a k^{2} / H_{0}^{2}}{a^{3}}-9\right) H_{0}^{2} \lambda+O\left(\lambda^{2}\right)$

In this case using the BBN constraint we get -8.9 . $10^{-29}<\lambda H_{0}^{2}<2.8 \cdot 10^{-28}$. In the general case, the allowed parameter space, shown in Fig. 2, is rather complicated. When $n>0$ then $\lambda$ is constrained to be $-2.8 \cdot 10^{-27}<\lambda H_{0}^{2}<-1.8 \cdot 10^{-27}$, while when $n<0$ we cannot give a bound for $\lambda$ in closed form.

It should be noted that while it seems that when we combine the two constraints there is no allowed parameter space at all for our model, there are actually values for $(n, \lambda)$ that satisfy both observational constraints, e.g. $(n, \lambda)=(-2 / 3,0.015873)$, but they require extreme fine-tuning to achieve agreement with the observations and some of these values exhibit unphysical behavior for $G_{\text {eff }}$ at early times, such as singularities. Therefore we may conclude that our simple toy model is in some stress with observations as the allowed parameter space is very small and even then for some of the allowed parameter values, the model may exhibit unphysical behavior. This of course does not mean that the whole class of theories with a nonminimal coupling is problematic, as one may well choose more suitable forms of the functions $f_{1}(R)$, $f_{2}(R)$ and $G\left(L_{m}\right)$ that satisfy both observational constraints.

\section{CONCLUSIONS}

We derived the matter density perturbation equation and the effective gravitational "constant" $G_{e f f}$ for the action (2.1). Our analysis covers $f(R)$ models with an arbitrary coupling of the matter content to gravity for any matter Lagrangian that depends on the fluid density $L_{m}=L_{m}\left(\rho_{m}\right)$. In order to recover the ideal pressureless fluid we chose $L_{m}=-\rho$. Having chosen some other dependence for the Lagrangian our results would be different since the equations of motion depend explicitly on the choice of the Lagrangian. As we have mentioned earlier, having more than one Lagrangian to describe the same fluid, eg $L_{m}=p$ or $L_{m}=-\rho$ for pressureless dust, is not a problem in GR as all choices will give the same equations of motion, but in theories with a nonminimal coupling, different choices in general will give different phenomenology.

We also evaluated two parameters $\eta$, which quantifies the strength of an anisotropic stress, and $\Sigma=q(1+\eta / 2)$, where $q$ quantifies the deviation of the gravitational constant today. These parameters can be used to constrain the theory with weak lensing observations and if such surveys measure deviations from GR, for which $\left.(\Sigma, \eta)\right|_{G R}=(1,0)$ then those two parameters could be used to differentiate between modified gravity and dark energy models.

Finally, we used a toy model with Lagrangian density $R / 2+(1+\lambda R) G\left(L_{m}\right)$, where $G\left(L_{m}\right)$ is given by $G\left(L_{m}\right)=A L_{m}^{n+1}$ and $L_{m}=-\rho$. This model has the energy-momentum tensor of pressureless dust, but exhibits nontrivial phenomenology compared to its counterpart from GR. We presented an analytical solution to the modified Friedmann equation and gave the scale factor $a$ in terms of the cosmic time $t$. However, our toy model is in some stress with observations as the allowed parameter space is very small. In order to have an old enough universe, so as to accommodate the oldest observed clusters, the required values for $\lambda$ are much larger than those allowed by the BBN constraints (see Figs. 1 

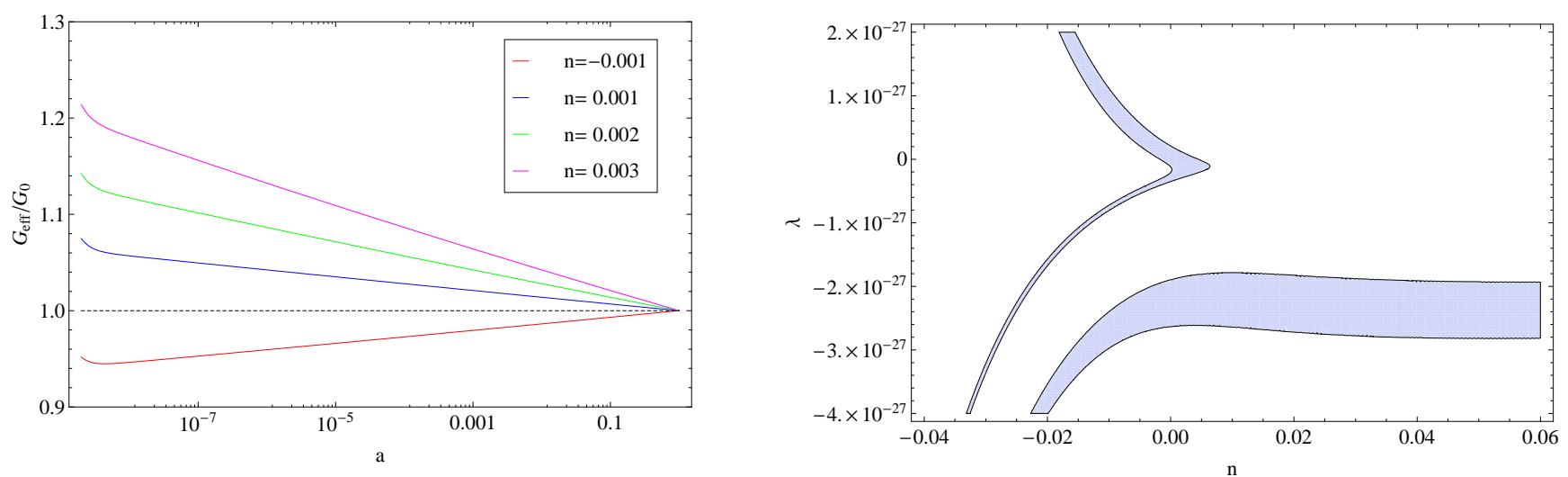

FIG. 2: Left: The evolution of the effective gravitational constant $G_{e f f} / G_{0}$ for $\lambda=10^{-29}$ and various values of $n$. Right: The allowed parameter space such that $\frac{\delta G_{e f f}}{G_{0}}$ is within the $1 \sigma$ of the nucleosynthesis bounds. $\left(\lambda\right.$ is in units of $\left.1 / H_{0}^{2}.\right)$

and 2). However, this fact should be interpreted with care since Ref. [41] used a simple modification of the Friedmann equation to derive the BBN constraints we used. A full analysis of the implications of modified gravity models with arbitrary coupling between matter and geometry during nucleosynthesis was beyond the scope of this analysis and is left for future work.

\section{Acknowledgements}

The author would like to thank H. Collins, T. Harko, T. Sotiriou and S. Tsujikawa for useful discussions. This work was supported by the Niels Bohr International
Academy and by the EU FP6 Marie Curie Research \& Training Network "UniverseNet" (MRTN-CT-2006035863).

\section{APPENDIX A: THE PERTURBATION EQUATIONS}

In this Appendix we have gathered some of the equations that if left in the main text they would make the analysis difficult to follow.

The Fourier transformed perturbation equations are given by

$$
\begin{aligned}
& -f_{2} K \delta \rho_{m}-3\left(\delta F_{1}+2 G \delta F_{2}\right) \dot{H}+6 F_{2} K \dot{H}\left(2 L_{m} \Phi+2 \rho_{m} \Phi-\delta \rho_{m} L_{m}^{\prime}\left(\rho_{m}\right)\right) \\
& -f_{2} \delta \rho_{m}\left(L_{m}+\rho_{m}\right) K^{\prime}\left(L_{m}\right) L_{m}^{\prime}\left(\rho_{m}\right)-3 H^{2}\left(\delta F_{1}+2\left(F_{1} \Phi+G\left(\delta F_{2}+2 F_{2} \Phi\right)+F_{2} K\left(-4\left(L_{m}+\rho_{m}\right) \Phi\right.\right.\right. \\
& \left.\left.\left.+\delta \rho_{m} L_{m}^{\prime}\left(\rho_{m}\right)\right)\right)\right)-3 \frac{k^{2}}{a^{2}} H^{2}\left(\delta F_{1}+2\left(-F_{1} \Psi+G\left(\delta F_{2}-2 F_{2} \Psi\right)+F_{2} K\left(\rho_{m}-L_{m}(\Phi-2 \Psi)\right.\right.\right. \\
& \left.\left.\left.+\delta \rho_{m} L_{m}^{\prime}\left(\rho_{m}\right)\right)\right)\right)-3\left(\dot{F}_{1}+2 G \dot{F}_{2}+2 F_{2} K L_{m}^{\prime}\left(\rho_{m}\right) \rho_{m}\right) \dot{\Psi}+6 F_{2} K\left(L_{m}+\rho_{m}\right) \ddot{\Psi} \\
& +3 H\left(-2 \Phi\left(\dot{F}_{1}+2 G \dot{F}_{2}+2 F_{2} K L_{m}^{\prime}\left(\rho_{m}\right) \rho_{m}\right)+2 F_{2} \delta \rho_{m} L_{m}^{\prime}\left(\rho_{m}\right)^{2} \dot{\rho}_{m} K^{\prime}\left(L_{m}\right)+\delta \dot{F}_{1}+2 G \delta \dot{F}_{2}-\right. \\
& 2\left(F_{1}+2 F_{2} G\right) \dot{\Psi}+2 K\left(\delta F_{2} L_{m}^{\prime}\left(\rho_{m}\right)+\delta \rho_{m}\left(\dot{F}_{2} L_{m}^{\prime}\left(\rho_{m}\right)+F_{2} \dot{\rho}_{m} L_{m}^{\prime \prime}\left(\rho_{m}\right)\right)\right. \\
& \left.\left.+F_{2}\left(L_{m}^{\prime}\left(\rho_{m}\right) \delta \dot{\rho}_{m}+\left(L_{m}+\rho_{m}\right)(\dot{\Phi}+4 \dot{\Psi})\right)\right)\right)=0
\end{aligned}
$$

$$
\Phi-\Psi+\frac{\delta F_{1}+2 G\left(L_{m}\right)\left((\Phi-\Psi) F_{2}+\delta F_{2}\right)+2 K\left(L_{m}\right) F_{2} \delta \rho_{m} L_{m}^{\prime}\left(\rho_{m}\right)}{F_{1}}=0
$$




$$
\begin{aligned}
& -\delta \dot{\rho}_{m}-3 H \delta \rho_{m}+\rho\left(3 \dot{\Psi}-\frac{k^{2}}{a} v_{m}\right)=\left(-6 a^{2}(\ddot{H}+4 H \dot{H}) F_{2} \delta f_{2}\left(L_{m}+\rho_{m}\right) K^{2}-f_{2}\left(2 F _ { 2 } \left(12 H^{2} \dot{\Phi}\left(L_{m}+\rho_{m}\right) a^{2}\right.\right.\right. \\
& +3\left(\left(L_{m}+\rho_{m}\right)\left(2 \Phi \ddot{H}+\dot{H}(3 \dot{\Phi}+4 \dot{\Psi})+\frac{\partial^{3} \Psi}{\partial t^{3}}\right)-\ddot{H} \delta \rho_{m}\left(L_{m}^{\prime}\left(\rho_{m}\right)+1\right)\right) a^{2}-k^{2}(\dot{\Phi}-2 \dot{\Psi})\left(L_{m}+\rho_{m}\right) \\
& +H\left(-12 \dot{H} \delta \rho_{m}\left(L_{m}^{\prime}\left(\rho_{m}\right)+1\right) a^{2}+\left(3(\ddot{\Phi}+4 \ddot{\Psi}) a^{2}-4 k^{2} \Psi+2 \Phi\left(12 \dot{H} a^{2}+k^{2}\right)\right) L_{m}+\left(3(\ddot{\Phi}+4 \ddot{\Psi}) a^{2}\right.\right. \\
& \left.\left.\left.\left.-4 k^{2} \Psi+2 \Phi\left(12 \dot{H} a^{2}+k^{2}\right)\right) \rho_{m}\right)\right)-a^{2} \delta \dot{f}_{2}\left(L_{m}+\rho_{m}\right)\right) K^{2}+a^{2} f_{2}^{2}\left(K \left(\delta \dot{\rho}_{m}\left(L_{m}+\rho_{m}\right) K^{\prime}\left(L_{m}\right) L_{m}^{\prime}\left(\rho_{m}\right)\right.\right. \\
& \left.+\dot{\rho_{m}} \delta \rho_{m}\left(\left(L_{m}+\rho_{m}\right) K^{\prime \prime}\left(L_{m}\right) L_{m}^{\prime}\left(\rho_{m}\right)^{2}+K^{\prime}\left(L_{m}\right)\left(L_{m}^{\prime}\left(\rho_{m}\right)^{2}+L_{m}^{\prime}\left(\rho_{m}\right)+\left(L_{m}+\rho_{m}\right) L_{m}^{\prime \prime}\left(\rho_{m}\right)\right)\right)\right) \\
& \left.\left.-\dot{\rho}_{m} \delta \rho_{m}\left(L_{m}+\rho_{m}\right) K^{\prime}\left(L_{m}\right)^{2} L_{m}^{\prime}\left(\rho_{m}\right)^{2}\right)\right) /\left(a^{2} K f_{2}^{2}\left(K+\delta \rho_{m} K^{\prime}\left(L_{m}\right) L_{m}^{\prime}\left(\rho_{m}\right)\right)\right)
\end{aligned}
$$

$$
\begin{aligned}
& \Phi \rho_{m}-a\left(\dot{\rho} v_{m}+4 H \rho_{m} v_{m}+\rho_{m} \dot{v}_{m}\right)=\left(-12 H^{3} K F_{2} \rho_{m} v_{m} a^{3}+6 H K\left(H^{2}+\dot{H}\right) F_{2} \rho_{m} v_{m} a^{3}\right. \\
& +\dot{\rho} f_{2} \rho_{m} v_{m} K^{\prime}\left(L_{m}\right) L_{m}^{\prime}\left(\rho_{m}\right) a^{3}+\left(K\left(L_{m} \delta f_{2}+6 a\left(H^{3}+3 \dot{H} H+\ddot{H}\right) F_{2} \rho_{m} v_{m}\right)\right. \\
& \left.\left.+f_{2} L_{m} \delta \rho_{m} K^{\prime}\left(L_{m}\right) L_{m}^{\prime}\left(\rho_{m}\right)\right) a^{2}\right) /\left(a^{2} f_{2}\left(K+\delta \rho_{m} K^{\prime}\left(L_{m}\right) L_{m}^{\prime}\left(\rho_{m}\right)\right)\right)
\end{aligned}
$$

Under the subhorizon approximation the differential

equation for the growth of perturbations $\delta$ is

$$
\begin{aligned}
& \ddot{\delta}_{m}+2 H \dot{\delta}_{m}+\frac{k^{2} \Phi}{a^{2}}-\frac{k^{2}}{a^{2}}\left(\left(L _ { m } ( K - \delta _ { m } \rho _ { m } K ^ { \prime } ( L _ { m } ) L _ { m } ^ { \prime } ( \rho _ { m } ) ) \left(f_{2} \delta_{m} \rho_{m} K^{\prime}\left(L_{m}\right) L_{m}^{\prime}\left(\rho_{m}\right) a^{2}\right.\right.\right. \\
& \left.\left.\left.+2 k^{2} K(\Phi-2 \Psi) F_{2}\right)\right) /\left(a^{2} K^{2} f_{2} \rho_{m}\right)\right) \simeq 0
\end{aligned}
$$

Using the subhorizon approximation in Eqs. (A1) and (A2), we get a coupled set of equations for $\Phi$ and $\Psi$ :

$$
\begin{aligned}
& \frac{1}{a^{4}}\left(-f_{2} \delta_{m} \rho_{m}\left(K+\left(L_{m}+\rho_{m}\right) K^{\prime}\left(L_{m}\right) L_{m}^{\prime}\left(\rho_{m}\right)\right) a^{4}\right. \\
& -2 k^{2}\left(\Psi F_{1}+F_{2}\left(2 G \Psi+K\left((\Phi-2 \Psi) L_{m}\right.\right.\right. \\
& \left.\left.+\rho_{m}\left(\Phi-2 \Psi-\delta_{m} L_{m}^{\prime}\left(\rho_{m}\right)\right)\right)\right) a^{2} \\
& \left.+2\left(F_{1, R}+2 F_{2, R} G\right) k^{4}(\Phi-2 \Psi)\right)=0
\end{aligned}
$$

$$
\begin{aligned}
& \left(\left((\Phi-\Psi) F_{1}+2 F_{2}\left(G(\Phi-\Psi)+K \delta_{m} \rho_{m} L_{m}^{\prime}\left(\rho_{m}\right)\right)\right) a^{2}\right. \\
& \left.+2\left(F_{1, R}+2 F_{2, R} G\right) k^{2}(\Phi-2 \Psi)\right) /\left(a^{2} F_{1}\right)=0 \quad(\mathrm{~A} 7)
\end{aligned}
$$

By solving the system of equations (A6) and (A7) for $\Phi$ and $\Psi$ we find

$$
\begin{aligned}
& \Psi=-\frac{a^{2}}{k^{2}} \delta_{m} \rho_{m}\left(f _ { 2 } ( F _ { 1 } + 2 G F _ { 2 } ) \left(K+\left(L_{m}+\rho_{m}\right)\right.\right. \\
& \left.K^{\prime}\left(L_{m}\right) L_{m}^{\prime}\left(\rho_{m}\right)\right) a^{2}+2 k^{2}\left(-K F_{2}\left(F_{1}+2 F_{2}(G\right.\right. \\
& \left.\left.+K\left(L_{m}+\rho_{m}\right)\right)\right) L_{m}^{\prime}\left(\rho_{m}\right)+\left(F_{1, R}+2 F_{2, R} G\right) f_{2} \\
& \left.\left.\left(K+\left(L_{m}+\rho_{m}\right) K^{\prime}\left(L_{m}\right) L_{m}^{\prime}\left(\rho_{m}\right)\right)\right)\right) / \\
& \left(2 ( F _ { 1 } + 2 G F _ { 2 } ) \left(\left(F_{1}+F_{2}\left(2 G-K\left(L_{m}+\rho_{m}\right)\right)\right) a^{2}\right.\right. \\
& \left.\left.+3\left(F_{1, R}+2 F_{2, R} G\right) k^{2}\right)\right)
\end{aligned}
$$

and

$$
\begin{aligned}
& \Phi=-\frac{a^{2}}{k^{2}} \delta_{m} \rho_{m}\left(f _ { 2 } ( F _ { 1 } + 2 G F _ { 2 } ) \left(K+\left(L_{m}+\rho_{m}\right)\right.\right. \\
& \left.K^{\prime}\left(L_{m}\right) L_{m}^{\prime}\left(\rho_{m}\right)\right) a^{2}+2 k^{2}\left(K F _ { 2 } \left(F_{1}+2 F_{2}(G-\right.\right. \\
& \left.\left.2 K\left(L_{m}+\rho_{m}\right)\right)\right) L_{m}^{\prime}\left(\rho_{m}\right)+2 F_{1, R} f_{2}\left(K+\left(L_{m}+\rho_{m}\right)\right. \\
& \left.K^{\prime}\left(L_{m}\right) L_{m}^{\prime}\left(\rho_{m}\right)\right)+4 F_{2, R} G f_{2}\left(K+\left(L_{m}+\rho_{m}\right)\right. \\
& \left.\left.\left.K^{\prime}\left(L_{m}\right) L_{m}^{\prime}\left(\rho_{m}\right)\right)\right)\right) /\left(2 ( F _ { 1 } + 2 G F _ { 2 } ) \left(\left(F_{1}+F_{2}(2 G-\right.\right.\right. \\
& \left.\left.\left.\left.K\left(L_{m}+\rho_{m}\right)\right)\right) a^{2}+3\left(F_{1, R}+2 F_{2, R} G\right) k^{2}\right)\right)
\end{aligned}
$$

By using (A9) to define a Poisson equation in the Fourier space we get $G_{\text {eff }}$

$$
\begin{aligned}
& G_{e f f}=\frac{1}{8 \pi}\left(f _ { 2 } ( F _ { 1 } + 2 G F _ { 2 } ) \left(K+\left(L_{m}+\rho_{m}\right)\right.\right. \\
& \left.K^{\prime}\left(L_{m}\right) L_{m}^{\prime}\left(\rho_{m}\right)\right) a^{2}+2 k^{2}\left(K F _ { 2 } \left(F_{1}+2 F_{2}(G-\right.\right. \\
& \left.\left.2 K\left(L_{m}+\rho_{m}\right)\right)\right) L_{m}^{\prime}\left(\rho_{m}\right)+2 F_{1, R} f_{2}\left(K+\left(L_{m}+\rho_{m}\right)\right. \\
& \left.K^{\prime}\left(L_{m}\right) L_{m}^{\prime}\left(\rho_{m}\right)\right)+4 F_{2, R} G f_{2}\left(K+\left(L_{m}+\rho_{m}\right)\right. \\
& \left.\left.\left.K^{\prime}\left(L_{m}\right) L_{m}^{\prime}\left(\rho_{m}\right)\right)\right)\right) /\left(( F _ { 1 } + 2 G F _ { 2 } ) \left(\left(F_{1}+F_{2}(2 G-\right.\right.\right. \\
& \left.\left.\left.\left.K\left(L_{m}+\rho_{m}\right)\right)\right) a^{2}+3\left(F_{1, R}+2 F_{2, R} G\right) k^{2}\right)\right)
\end{aligned}
$$

The parameter $\beta$, which corresponds to the last term 
of Eq. (A5), after using Eqs. (A8) and (A9), is given by

$$
\begin{aligned}
& \beta=\frac{1}{4 \pi} \frac{k^{2}}{a^{2}} L_{m}\left(f _ { 2 } \left(F_{2}\left(K^{2}+2 G K^{\prime}\left(L_{m}\right) L_{m}^{\prime}\left(\rho_{m}\right)\right) a^{2}\right.\right. \\
& \left.+\left(F_{1} a^{2}+3\left(F_{1, R}+2 F_{2, R} G\right) k^{2}\right) K^{\prime}\left(L_{m}\right) L_{m}^{\prime}\left(\rho_{m}\right)\right) \\
& \left.\left.-6 k^{2} K^{2} F_{2}^{2} L_{m}^{\prime}\left(\rho_{m}\right)\right)\right) /\left(K f _ { 2 } \rho _ { m } \left(-F_{1} a^{2}\right.\right. \\
& +F_{2}\left(-2 G+K L_{m}+K \rho_{m}\right) a^{2} \\
& \left.\left.-3\left(F_{1, R}+2 F_{2, R} G\right) k^{2}\right)\right)
\end{aligned}
$$

The two parameters $\eta$ and $q$ defined in the main text are given by

$$
\begin{aligned}
& \eta=2 \frac{k^{2}}{a^{2}}\left(2 K F_{2}\left(F_{1}+F_{2}\left(2 G-K L_{m}-K \rho_{m}\right)\right) L_{m}^{\prime}\left(\rho_{m}\right)\right. \\
& +\left(F_{1, R}+2 F_{2, R} G\right) f_{2}\left(K+L_{m} K^{\prime}\left(L_{m}\right) L_{m}^{\prime}\left(\rho_{m}\right)\right. \\
& \left.\left.+\rho_{m} K^{\prime}\left(L_{m}\right) L_{m}^{\prime}\left(\rho_{m}\right)\right)\right) /\left(f _ { 2 } ( F _ { 1 } + 2 G F _ { 2 } ) \left(K+\left(L_{m}+\rho_{m}\right)\right.\right. \\
& \left.K^{\prime}\left(L_{m}\right) L_{m}^{\prime}\left(\rho_{m}\right)\right)+2 k^{2} / a^{2}\left(\left(F_{1, R}+2 F_{2, R} G\right) f_{2}(K\right. \\
& \left.+L_{m} K^{\prime}\left(L_{m}\right) L_{m}^{\prime}\left(\rho_{m}\right)+\rho_{m} K^{\prime}\left(L_{m}\right) L_{m}^{\prime}\left(\rho_{m}\right)\right) \\
& \left.\left.-K F_{2}\left(F_{1}+2 F_{2}\left(G+K L_{m}+K \rho_{m}\right)\right) L_{m}^{\prime}\left(\rho_{m}\right)\right)\right) \quad(\mathrm{A} 12)
\end{aligned}
$$

and

$$
\begin{aligned}
& q=\frac{1}{8 \pi G_{0}}\left(f _ { 2 } ( F _ { 1 } + 2 G F _ { 2 } ) \left(K+\left(L_{m}+\rho_{m}\right)\right.\right. \\
& \left.K^{\prime}\left(L_{m}\right) L_{m}^{\prime}\left(\rho_{m}\right)\right) a^{2}+2 k^{2}\left(-K F_{2}\left(F_{1}+2 F_{2}(G\right.\right. \\
& \left.\left.+K\left(L_{m}+\rho_{m}\right)\right)\right) L_{m}^{\prime}\left(\rho_{m}\right)+F_{1, R} f_{2}\left(K+\left(L_{m}+\rho_{m}\right)\right. \\
& \left.K^{\prime}\left(L_{m}\right) L_{m}^{\prime}\left(\rho_{m}\right)\right)+2 F_{2, R} G f_{2}\left(K+\left(L_{m}+\rho_{m}\right)\right. \\
& \left.\left.\left.K^{\prime}\left(L_{m}\right) L_{m}^{\prime}\left(\rho_{m}\right)\right)\right)\right) /\left(( F _ { 1 } + 2 G F _ { 2 } ) \left(\left(F_{1}+F_{2}(2 G-\right.\right.\right. \\
& \left.\left.\left.\left.K\left(L_{m}+\rho_{m}\right)\right)\right) a^{2}+3\left(F_{1, R}+2 F_{2, R} G\right) k^{2}\right)\right)
\end{aligned}
$$

The explicit form of $\Sigma$, used to confront the modified gravity models with weak lensing observations, is given by

$$
\begin{aligned}
& \Sigma=\left(f_{2}\left(F_{1}+2 G F_{2}\right)\left(K+\left(L_{m}+\rho_{m}\right) K^{\prime}\left(L_{m}\right) L_{m}^{\prime}\left(\rho_{m}\right)\right) a^{2}\right. \\
& +3 k^{2}\left(\left(F_{1, R}+2 F_{2, R} G\right) K f_{2}-\left(L_{m}+\rho_{m}\right)\right. \\
& \left.\left.\left(2 K^{2} F_{2}^{2}-\left(F_{1, R}+2 F_{2, R} G\right) f_{2} K^{\prime}\left(L_{m}\right)\right) L_{m}^{\prime}\left(\rho_{m}\right)\right)\right) / \\
& \left(8 G _ { 0 } \pi ( F _ { 1 } + 2 G F _ { 2 } ) \left(\left(F_{1}+F_{2}\left(2 G-K\left(L_{m}+\rho_{m}\right)\right)\right) a^{2}\right.\right. \\
& \left.\left.+3\left(F_{1, R}+2 F_{2, R} G\right) k^{2}\right)\right)
\end{aligned}
$$

In the case of our toy model of Sec. IV, $G_{e f f}$ can be calculated by using Eq. (A10) and is given by

$$
\begin{aligned}
G_{e f f}(a)= & G_{0}\left(a ( 1 8 ( n + 1 ) \lambda H _ { 0 } ^ { 2 } - 1 ) \left(-3 \lambda\left(9 a^{2}(n+1)-8 k^{2}(3 n+2) \lambda\right) H_{0}^{2}\left(6(3 n+2) \lambda H_{0}^{2}-1\right) a^{3(n+1)}+\right.\right. \\
& \left.\left.\left(a^{2}-2 k^{2} \lambda\right)\left(1-6(3 n+2) \lambda H_{0}^{2}\right)^{2} a^{6 n+6}+36(3 n+2) \lambda^{2}\left(a^{2}(3 n+4)-2 k^{2}(3 n+2) \lambda\right) H_{0}^{4}\right)\right) / \\
& \left(\left(\left(6(3 n+2) \lambda H_{0}^{2}-1\right) a^{3 n+3}+6 \lambda H_{0}^{2}\right)\left(a^{3 n+3}\left(6(3 n+2) \lambda H_{0}^{2}-1\right)-6(3 n+2) \lambda H_{0}^{2}\right)^{2}\right. \\
& \left.\left(1+\lambda\left(-\frac{2 k^{2}}{H_{0}^{2}}+54(n+1)(3 n+2) \lambda H_{0}^{2}-9 n+3\right) H_{0}^{2}\right)\right)
\end{aligned}
$$

[1] A. G. Riess et al., Astron. J. 116, 1009 (1998); Astron. J. 117,707 (1999); P. Astier et al., Astron. Astrophys. 447, 31 (2006); G. Miknaitis et al., Astrophys. J. 666, 674 (2007);

[2] E. J. Copeland, M. Sami and S. Tsujikawa, Int. J. Mod. Phys. D 15, 1753 (2006) arXiv:hep-th/0603057.

[3] L. Perivolaropoulos, AIP Conf. Proc. 848, 698 (2006) arXiv:astro-ph/0601014.

[4] E. Komatsu et al. [WMAP Collaboration], arXiv:0803.0547 [astro-ph].

[5] L. Perivolaropoulos, arXiv:0811.4684 [astro-ph].
[6] L. Perivolaropoulos and A. Shafieloo, arXiv:0811.2802 [astro-ph].

[7] Y. Fujii, Phys. Rev. D 26, 2580 (1982); L. H. Ford, Phys. Rev. D 35, 2339 (1987); C. Wetterich, Nucl. Phys B. 302, 668 (1988); B. Ratra and J. Peebles, Phys. Rev D 37, 3406 (1988); Y. Fujii and T. Nishioka, Phys. Rev. D 42, 361 (1990); E. J. Copeland, A. R. Liddle, and D. Wands, Ann. N. Y. Acad. Sci. 688, 647 (1993); C. Wetterich, A\&A 301, 321 (1995); P. G. Ferreira and M. Joyce, Phys. Rev. Lett. 79, 4740 (1997); Phys. Rev. D 58, 023503 (1998); R. R. Cald- 
well, R. Dave and P. J. Steinhardt, Phys. Rev. Lett. 80, 1582 (1998); I. Zlatev, L. M. Wang and P. J. Steinhardt, Phys. Rev. Lett. 82, 896 (1999); P. J. Steinhardt, L. M. Wang and I. Zlatev, Phys. Rev. D 59, 123504 (1999); L. Perivolaropoulos, Phys. Rev. D 71, 063503 (2005) arXiv:astro-ph/0412308.

[8] N. Bilic, G. B. Tupper and R. D. Viollier, Phys. Lett. B 535, 17 (2002) arXiv:astro-ph/0111325; M. C. Bento, O. Bertolami and A. A. Sen, Phys. Rev. D 66, 043507 (2002) arXiv:gr-qc/0202064.

[9] A. Friedland, H. Murayama and M. Perelstein, Phys. Rev. D 67, 043519 (2003) arXiv:astro-ph/0205520.

[10] S. Nojiri and S. D. Odintsov, Gen. Rel. Grav. 38, 1285 (2006) arXiv:hep-th/0506212.

[11] B. Boisseau, G. Esposito-Farèse, D. Polarski and A. A. Starobinsky, Phys. Rev. Lett. 85, 2236 (2000); G. Esposito-Farèse and D. Polarski, Phys. Rev. D 63063504 (2001);

[12] J. P. Uzan, Phys. Rev. D 59, $123510 \quad$ (1999) arXiv:gr-qc/9903004; Y. Fujii, Phys. Rev. D62, 044011 (1999); N. Bartolo and M. Pietroni, Phys. Rev. D 61 023518 (1999); F. Perrotta, C. Baccigalupi and S. Matarrese, Phys. Rev. D 61, 023507 (2000); D. F. Torres, Phys. Rev. D 66, 043522 (2002); R. Gannouji, D. Polarski, A. Ranquet and A. A. Starobinsky, JCAP 0609, 016 (2006); S. Capozziello, S. Nojiri and S. D. Odintsov, Phys. Lett. B 634, 93 (2006) arXiv:hep-th/0512118; A. Riazuelo and J. P. Uzan, Phys. Rev. D 66, 023525 (2002) arXiv:astro-ph/0107386.

[13] S. Capozziello, S. Nesseris and L. Perivolaropoulos, JCAP 0712, 009 (2007) arXiv:0705.3586 [astro-ph]].

[14] S. Nojiri and S. D. Odintsov, Gen. Rel. Grav. 36, 1765 (2004); M. E. Soussa and R. P. Woodard, Gen. Rel. Grav. 36, 855 (2004); G. Allemandi, A. Borowiec and M. Francaviglia, Phys. Rev. D 70, 103503 (2004); D. A. Easson, Int. J. Mod. Phys. A 19, 5343 (2004); S. M. Carroll, A. De Felice, V. Duvvuri, D. A. Easson, M. Trodden and M. S. Turner, Phys. Rev. D 71, 063513 (2005); S. Carloni, P. K. S. Dunsby, S. Capozziello and A. Troisi, Class. Quant. Grav. 22, 4839 (2005); S. Capozziello, V. F. Cardone and A. Troisi, Phys. Rev. D 71, 043503 (2005); G. Cognola, E. Elizalde, S. Nojiri, S. D. Odintsov and S. Zerbini, JCAP 0502, 010 (2005); A.F. Zakharov et al., Phys. Rev. D 74, 107101 (2006); J. A. R. Cembranos, Phys. Rev. D 73, 064029 (2006) arXiv:gr-qc/0507039.

[15] R. Maartens, Living Rev. Rel. 7, 7 (2004) arXiv:gr-qc/0312059; V. Sahni and Y. Shtanov, JCAP 0311, 014 (2003) arXiv:astro-ph/0202346; G. Kofinas, G. Panotopoulos and T. N. Tomaras, JHEP 0601, 107 (2006) arXiv:hep-th/0510207; C. Bogdanos, A. Dimitriadis and K. Tamvakis, Phys. Rev. D 75, 087303 (2007).

[16] S. Nojiri and S. D. Odintsov, Phys. Rev. D 68, 123512 (2003) arXiv:hep-th/0307288.
[17] S. Nojiri and S. D. Odintsov, arXiv:0807.0685 [hep-th].

[18] S. Nojiri and S. D. Odintsov, eConf C0602061, 06 (2006) [Int. J. Geom. Meth. Mod. Phys. 4, 115 (2007)] arXiv:hep-th/0601213.

[19] T. P. Sotiriou, arXiv:0810.5594 [gr-qc].

[20] T. P. Sotiriou and V. Faraoni, arXiv:0805.1726 [gr-qc].

[21] N. D. Birrell, P. C. W. Davies, Quantum Fields in Curved Space, Cambridge University Press, Cambridge (UK) (1982); I. L. Buchbinder, S. D. Odintsov and I. L. Shapiro, Effective Action in Quantum Gravity, IOP, Bristol/Philadelphia (1992).

[22] S. D. Odintsov, S. Nojiri, Phys. Lett. B 576, 5 (2003).

[23] G. Allemandi, A. Borowiec, M. Francaviglia and S. D. Odintsov, Phys. Rev. D 72, 063505 (2005) arXiv:gr-qc/0504057.

[24] T. Inagaki, S. Nojiri and S. D. Odintsov, JCAP 0506, 010 (2005) arXiv:gr-qc/0504054.

[25] O. Bertolami, C. G. Boehmer, T. Harko and F. S. N. Lobo, Phys. Rev. D 75, 104016 (2007) arXiv:0704.1733 [gr-qc]].

[26] S. Nojiri and S. D. Odintsov, Phys. Lett. B 599, 137 (2004) arXiv:astro-ph/0403622.

[27] T. P. Sotiriou and V. Faraoni, Class. Quant. Grav. 25, 205002 (2008) arXiv:0805.1249 [gr-qc]].

[28] T. P. Sotiriou, Phys. Lett. B 664, 225 (2008) arXiv:0805.1160 [gr-qc]].

[29] O. Bertolami, T. Harko, F. S. N. Lobo and J. Paramos, arXiv:0811.2876 [gr-qc].

[30] O. Bertolami, F. S. N. Lobo and J. Paramos, Phys. Rev. D 78, 064036 (2008) arXiv:0806.4434 [gr-qc]].

[31] T. Harko, Phys. Lett. B 669, 376 (2008) arXiv:0810.0742 [gr-qc]].

[32] S. Nesseris and L. Perivolaropoulos, Phys. Rev. D 77, 023504 (2008) arXiv:0710.1092 [astro-ph]].

[33] S. Nesseris and L. Perivolaropoulos, JCAP 0701, 018 (2007) arXiv:astro-ph/0610092.

[34] L. Amendola, M. Kunz and D. Sapone, JCAP 0804, 013 (2008) arXiv:0704.2421 [astro-ph]].

[35] S. Tsujikawa, Phys. Rev. D 76, 023514 (2007) arXiv:0705.1032 [astro-ph]].

[36] T. Koivisto, Class. Quant. Grav. 23, 4289 (2006) arXiv:gr-qc/0505128.

[37] S. Carloni, P. K. S. Dunsby and A. Troisi, Phys. Rev. D 77, 024024 (2008) arXiv:0707.0106 [gr-qc]].

[38] K. N. Ananda, S. Carloni and P. K. S. Dunsby, arXiv:0809.3673 [astro-ph].

[39] C. Schimd, J. P. Uzan and A. Riazuelo, Phys. Rev. D 71, 083512 (2005) arXiv:astro-ph/0412120.

[40] R. G. Gratton, F. Fusi Pecci, E. Carretta, G. Clementini, C. E. Corsi and M. Lattanzi, Astrophys. J. 491, 749 (1997) arXiv:astro-ph/9704150.

[41] C. Bambi, M. Giannotti and F. L. Villante, Phys. Rev. D 71, 123524 (2005) arXiv:astro-ph/0503502. 